\section{Scale for retrospective assessment of immediate concussion symptoms}

\section{Zack Zdenek Cernovsky, Paul Victor Fayez Istasy, Yves Bureau, Simon Chiu University of Western Ontario, London, Ontario, Canada}

\begin{abstract}
The retrospective diagnosis of concussion is often missed by clinicians. We present a brief scale for retrospective assessment of the immediate concussion symptoms (ICS) to facilitate the diagnosis of patients without visible head injury or full loss of consciousness. We administered the scale to 90 survivors of car accidents (mean age $42.0, \mathrm{SD}=13.6 ; 33$ males, 57 females) at 2 to 33 months after their accident. Our scale consists of 6 items and these were endorsed by the following \% of our respondents: feeling dazed $(64.4 \%$ of our 90 respondents), stunned $(73.3 \%)$, confused $(70.0 \%)$, disoriented $(62.2 \%)$, dizzy $(57.8 \%)$, and loss of consciousness $(22.2 \%)$. The statistical properties of the scale are satisfactory (Cronbach alpha = 0.74). The scale correlates with post-accident insomnia $(\mathrm{r}=0.28)$, depression $(r=0.29)$, and also with Rivermead measure of the chronic post-concussion syndrome $(\mathrm{r}=0.34)$. The ICS scale could be used as a starting point in longitudinal research with brain imaging procedures to evaluate the stages of recovery from the initial concussion. Attached are the English, Spanish, French, German, Italian, Russian, and Czech versions of our scale.
\end{abstract}

\section{Introduction}

The diagnosis of concussion is often missed in clinical work, especially by emergency physicians who have neither time nor an easy scale with which to rapidly evaluate more subtle aspects of concussion, especially if the patient appears still somewhat functional. The concept of a partial rather than complete loss of consciousness is important in the assessment of concussion. In the English language, a partial loss of consciousness or a pathological lowering of the level of consciousness can be represented by adjectives such as feeling dazed, stunned, disoriented, confused, or dizzy. The Glasgow Coma Scale would help to assess the concomitant physical signs, however, physicians are usually not present to assess such signs within the first minutes after the trauma to the brain. The person who sustained the trauma, but has not lost consciousness completely, is usually able to retrospectively recall how he or she subjectively felt at the time. Such information is subsequently useful for retrospective medical assessments.

Modern research indeed suggests that cerebral damage in concussions occurs even in those who did not fully lose consciousness and still appear able to perform at least some simple tasks. The neuropathologicalhistological research by Bennet Omalu1,2 and of Mez et al. ${ }^{3}$ documented that cerebral concussions occur even with relatively minor impacts such as in football players, impacts without a visible external head injury and very often also without a full loss of consciousness. Axonal shearing or rupture of blood vessels is often noted in brain areas between the white and the grey matter as these tissues slide over each other due to their different density (weight per $\mathrm{cm}^{3}$ ), particularly with the rotational movement of the head. ${ }^{4}$ The person can sustain cerebral damage without losing consciousness. The related immediate concussion symptoms and subsequent long-term psychopathological sequelae are observed very frequently in survivors of motor vehicle accidents (MVAs). We designed a scale to retrospectively measure the immediate symptoms of cerebral concussion.

\section{Materials and Methods}

Our Immediate Concussion Symptoms (ICS) scale was developed to quantify the relatively subtle initial symptoms of cerebral concussion as they occur within the first minutes after the MVA. Our scale (see Appendix 1) consists of 6 items. The first 5 assess feeling dazed, stunned, confused, disoriented, and dizzy and each of these is scored separately as a dichotomy (symptom absent $=0$, present $=1$ ). If the patient lost consciousness, all these first 5 items are scored on the basis of symptoms as they occurred within the very first minutes upon regaining consciousness. The last item is the loss of consciousness, scored as follows: absent $=0$, not certain $=1$, brief loss of consciousness $=2$, loss of consciousness lasting more than 5 minutes $=3$. Some persons are unable to retrospectively determine if they really lost consciousness because they have a related amnesia or also because the loss of consciousness, rather than being a distinct dichotomy, can be conceived as a dimension ranging from a partial, minor, or subtle to a more distinct impairment. The total score on the ICS scale is calculated by
Correspondence: Zack Cernovsky, Professor of Psychiatry, University of Western Ontario, 98 Greenbrier Crescent, London, Ontario, N6J $3 \times 9$ Canada.

Tel./Fax: +1.519.4718049.

E-mail: zcernovs@uwo.ca

Key words: concussion, post-concussion syndrome, Rivermead scale, traumatic brain injury.

Contributions: ZC, Interviews with the patients, Development of the scale, Statistical Analyses, Preparation of the manuscript; PI, Preparation of the data set, Conceptual analysis, Consultations on the scale with experts and clinicians at World Congress of Psychiatry: YB, Neuropsychological, statistical, and conceptual review of the manuscript; $\mathrm{SC}$, Conceptual psychiatric review of the scale and of the manuscript.

Conflict of interest: declare no potential conflict of interest.

Funding: none.

Conference presentation: This manuscript is an expanded version of our paper presented at the World Congress of Psychiatry, Mexico City, Mexico, September 27-30, 2018.

Received for publication: 7 October 2018. Revision received: 5 December 2018. Accepted for publication: 5 December 2018.

This work is licensed under a Creative Commons Attribution-NonCommercial 4.0 International License (CC BY-NC 4.0).

(C) Copyright Z.Z. Cernovsky et al., 2018

Licensee PAGEPress, Italy

Mental Illness 2018; 10:7901

doi:10.4081/mi.2018.7901

adding the points on the 6 items: the maximum score is 8 points.

Our sample included 90 patients (mean age 42.0, $\mathrm{SD}=13.6$; 33 males, 57 females) who were applying for compensation with their car insurer after an MVA and provided valid responses to our Immediate Concussion Symptoms (ICS) scale. The patients also completed the Brief Pain Inventory, ${ }^{5}$ Insomnia Severity Index, ${ }^{6}$ Items 10 to 12 of the Whiplash Disability Questionnaire, ${ }^{7}$ and the full Rivermead Post-Concussion Symptoms Questionnaire. ${ }^{8}$ The Rivermead scale measures the post-concussion syndrome as evaluated weeks or even years after the MVA, as the long-term neuropsychological syndrome. 


\section{Results}

The 6 items operationalizing the immediate concussion symptoms following the collisions were endorsed by the following proportions of our patients: feeling dazed (64.4\% of our 90 respondents), stunned $(73.3 \%)$, confused $(70.0 \%)$, disoriented $(62.2 \%)$, and dizzy $(57.8 \%)$. A brief loss of consciousness was reported by $21.1 \%$, loss lasting more than 5 minutes was reported by one person, and $13.3 \%$ were uncertain if they lost consciousness. The majority ( 58 of the 90 persons, i.e., $64.4 \%$ ) retrospectively assumed that they remained conscious.

The total scores on ICS scale ranged from $0(6.7 \%$ of respondents $)$ to $7(15.6 \%$ of respondents), with the average at 3.9 $(\mathrm{SD}=2.2)$. The Cronbach alpha coefficient of internal consistency was 0.74 , i.e., acceptable/fair. The item total correlations (with the item of interest always removed from the total) were all significant, ranging from 0.34 (for loss of consciousness) to 0.65 (for feeling disoriented).

Our ICS scale significantly correlated with the later post-concussion syndrome as measured via Rivermead (Pearson $r=0.34$ ), and also with post-MVA depressive mood (Item 10 from the Whiplash Disability Questionnaire, $\quad r=0.29$ ) and Insomnia Severity Index $(r=0.28)$, but not with age, gender, number of previous MVAs, and with measures of MVA related chronic pain (Items 3 to 5 of the Brief Pain Inventory) and of post-MVA anxiety or anger/irritability (Items 11 and 12 of the Whiplash Disability Questionnaire). The number of weeks since the MVA ranged from 8 to 142 (mean at 54.2, $\mathrm{SD}=32.2$ ), but was not significantly correlated to the ICS scale ( $\mathrm{r}=-$ 0.09).

\section{Discussion}

The ICS scale has satisfactory internal consistency and item total correlations. Its significant correlations with subsequent insomnia, depressive mood, and with scores on Rivermead measure of the post-concussion syndrome support its construct validity.

The ICS scale was developed to retrospectively assess the immediate symptoms of cerebral concussions, particularly for cases in which the person may still appear as somewhat functional, but could in fact experience some related consequences over subsequent weeks or even years. The ICS scale could be used as a starting point in longitudinal research with modern brain imaging procedures to evaluate the stages of recovery from the initial concussion. In theory, much damage can occur within the days after the impact to head due to toxic substances secreted by the torn axons: these toxic chemicals kill surrounding healthy neurons.

Questionnaire studies intrinsically rely on the weak premise that most English speakers interpret the relatively frequent words in approximately the same way. Our sample included only native English speakers with adequate education in order to reduce the likelihood of linguistic misinterpretations. The primary semantic connotation of the words "stunned" and "dazed" evokes the sense of being unable to think clearly or feeling too confused to react properly. Many dictionaries treat "stunned," "dazed," and also "confused" as synonyms. Only in their much less common usage as a metaphor are these concepts stretched to convey the concept of "surprise" or "shock," similarly as in "thunderstruck." Our concussion scale was administered to patients in the context of car accident that caused them long term pain: at the time of the interview, our patients' ratings of worst pain (item 3 of the Brief Pain Inventory) ranged from 2 to 10 on the scale from $0=$ "no pain," $10=$ "pain as bad as you can imagine." Although this particular physical situational context is more likely to imply a concussion, low total scores on our concussion scale such as those below 3 points (e.g., when only items "stunned" and "dazed" are endorsed) should be interpreted only with great caution.

The Appendix contains the English version of the ICS scale and its translations into several languages other than English, to be used as research tools, especially in clinical settings.

\section{Conclusions}

This brief scale for retrospective assess- ment of the immediate concussion symptoms allows physicians or psychologists to diagnose the subtle aspects of concussion, those that are usually missed by busy clinicians while interviewing patients who did not sustain a visible head injury or report no full loss of consciousness.

If used in research, the scale appears to be a logical starting point that provides the missing information on initial symptoms that precede the post-concussion syndrome as usually assessed via the Rivermead scale.

\section{References}

1. Omalu BI, DeKosky ST, Minster RL, et al. Chronic traumatic encephalopathy in a National Football League player. Neurosurgery 2005;57:128-34.

2. Omalu BI, DeKosky ST, Hamilton RL, et al. Chronic traumatic encephalopathy in a National Football League player: Part II. Neurosurgery 2006;59:1086-92

3. Mez J, Daneshvar DH, Kiernan PT, et al. Clinicopathological Evaluation of Chronic Traumatic Encephalopathy in Players of American Football. JAMA 2017;318:360-70.

4. Jang SH, Kwon YH. A Review of Traumatic Axonal Injury following Whiplash Injury As Demonstrated by Diffusion Tensor Tractography. Front Neurol 2018;9:500057

5. Cleeland CS, Ryan KM. Pain assessment: global use of the Brief Pain Inventory. Ann Acad Med Singapore 1994;23:129-38.

6. Morin CM, Belleville G, Bélanger L, Ivers $H$. The insomnia severity index: psychometric indicators to detect insomnia cases and evaluate treatment response. Sleep 2011;34:601-8.

7. Pinfold M, Niere KR, O'Leary EF, et al. Validity and internal consistency of a whiplash-specific disability measure. Spine (Phila Pa 1976). 2004;29:263-8.

8. Eyres S, Carey A, Gilworth G, et al. Construct validity and reliability of the Rivermead Post-Concussion Symptoms Questionnaire. Clin Rehabil 2005;19:878-87. 